\title{
Réponses de la filière des oléagineux en matière de traçabilité
}

Jean-Jacques PAPI

Cargill, Grains \& Oilseeds Related Businesses

Europe, Product Assurance Team,

18, rue des Gaudines,

78100 Saint Germain-en-Laye, France

<jean-jacques_papi@cargill.com>

\begin{abstract}
With regards to traceability in the oilseeds supply chain, the European regulatory environment must be considered, and particularly the Regulation (EC) N ${ }^{\circ} 178 / 2002$, published on January 28, 2004, laying down the traceability requirement and obligations. Traceability must be seen as a risk management tool, which is meant to ensure that targeted and accurate withdrawals or recalls of unsafe products can be undertaken. Oilseeds operators in Europe did not wait for the publication of the above-mentioned regulation to enhance systems that ensure traceability. The "one step back - one step forward" approach was already in place within the chain. Nevertheless, those oilseeds operators must ensure that the current systems set up are respectful with obligations as defined in European law (types of information, delay of archives, types of operators...).
\end{abstract}

Key words: traceability, regulation (EC) N¹78/2002, risk management tool

Ce document a pour objet d'aider tous les acteurs de la chaîne alimentaire à mieux comprendre ce règlement et à l'appliquer de manière correcte et uniforme. II donne notamment des orientations concernant la mise en œuvre de la traçabilité.

\section{Les produits et les opérateurs concernés par la traçabilité}

Les produits soumis à l'obligation de traçabilité :

- les denrées alimentaires et toute substance destinée à ou susceptible de leur être incorporée (additifs, arômes, auxiliaire technologique...);

- les aliments pour animaux et toute substance destinée à ou susceptible de leur être incorporée (additifs, arômes, auxiliaire technologique...) ;

- les animaux producteurs de denrées alimentaires.

Ne sont pas concernés : les semences, médicaments vétérinaires, produits phytosanitaires ou les matériaux destinés au contact des denrées alimentaires.

Les exploitants soumis à cette obligation : toutes les étapes de la chaîne alimentaire, depuis et y compris l'importation et la production primaire (animaux producteurs de denrées alimentaires, récoltes), jusqu'à la distribution, en passant par la production, la fabrication et la transformation des denrées alimentaires/ aliments pour animaux ainsi que leur transport et stockage.

\section{Les obligations des exploitants}

L'article 18 n'impose aucune obligation de moyens, mais il exige une obligation de résultats. Ainsi, les exploitants ont l'entière responsabilité du choix des systèmes de traçabilité, qu'ils déterminent en fonction d'une évaluation des risques et de leurs contraintes économiques.
Les obligations, a minima, qui s'imposent aux exploitants sont les suivantes:

- être en mesure d'identifier leurs fournisseurs et leurs entreprises clientes ;

- disposer « de systèmes et procédures permettant de mettre ces informations à la disposition des autorités compétentes, à la demande de celles-ci » ;

- étiqueter ou identifier de façon adéquate les denrées alimentaires ou les aliments pour animaux mis sur le marché, pour faciliter leur traçabilité.

Durée de conservation des données de traçabilité : dans le cas général, ces données sont à conserver pendant 5 ans. Le délai court à partir de la date de fabrication du produit. Ce délai nécessite des adaptations (par exemple, si produit à date de conservation, il s'agira alors de DLUO + 6 mois).

\section{Réponses des opérateurs de la filière des oléagineux en matière de traçabilité}

La traçabilité n'assure pas en soi la sécurité d'un aliment ; elle doit être appréhendée comme un outil ou un instrument de gestion de risques, à utiliser pour endiguer plus facilement un problème de sécurité alimentaire. Ainsi, la traçabilité doit permettre aux autorités compétentes de pouvoir procéder à des retraits ou à des rappels ciblés et exacts.

L'article 18 du règlement (CE) N No1782002 exige des opérateurs de la filière des oléagineux qu'ils soient capables d'identifier le ou les fournisseur(s) direct(s) de leurs produits, et le ou les client((s) direct(s) ayant acheté ces produits : principe du « juste avant » et du « juste après ». II est à noter que les exploitants du secteur alimentaire $n^{\prime}$ ont pas à identifier les clients directs lorsque ceux-ci sont les consommateurs finals. 
Le règlement n'oblige pas les opérateurs à établir un lien entre produits « entrants» et produits «sortants» ( traçabilité interne »). Néanmoins, cette traçabilité interne est recommandée car elle permettra des retraits plus ciblés et plus précis. Elle devrait permettre également de limiter la taille des lots incriminés dans le cas où un problème de sécurité alimentaire serait avéré.

Les actions en place chez la plupart des opérateurs de la filière des oléagineux depuis de nombreuses années (utilisation de systèmes propres de gestion des flux, des stocks...) permettent de respecter les obligations de traçabilité telles que définies dans la réglementation européenne :

- L'identification des fournisseurs et des clients pour les différents flux de produits (graines oléagineuses, huiles, tourteaux...) est la base des transactions commerciales. Une attention particulière doit cependant être apportée pour les imports en provenance de pays tiers car l'exigence de traçabilité s'applique à l'importateur (et non aux exploitants des pays tiers).

- De la même façon, l'ensemble des informations concernant la traçabilité sont disponibles, et sont souvent conservées plus que nécessaire. Conformément à la législation en vigueur, les informations à conserver et à mettre à disposition des autorités compétentes peuvent être classées en deux catégories, selon leur degré de priorité :

- de la première catégorie, relèvent toutes les informations dont les autorités compétentes doivent toujours pouvoir disposer (immédiatement) : nom et adresse du fournisseur ; nature des produits fournis par ce dernier; nom et adresse du client, nature des produits livrés à ce dernier ; date de transaction et de livraison ;

- de la deuxième catégorie, relèvent les informations supplémentaires dont la conservation est fortement recommandée (dans les plus brefs délais) : volume ou quantité ; numéro de lot, s'il y a lieu ou description du produit, du moyen de transport.

\section{La traçabilité au service de la sécurité alimentaire}

Si l'article 18 du règlement CE $n^{\circ} 178 / 2002$ constitue la pierre angulaire de la nouvelle législation concernant la sécurité alimentaire, d'autres articles du même règlement (art.19 et 20, notification des non-conformités), ainsi que de nouveaux textes législatifs parus en complément de ce document, notamment les règlements sur l'hygiène des produits: (CE) $N^{\circ} 852 / 2004$ et (CE) No $853 / 2004$, sont à considérer.

Les règlements «Hygiène» mentionnent I'application des principes de I'HACCP (reprenant les principes du Codex Alimentarius) et encouragent au développement des guides de bonnes pratiques hygiéniques au niveau national et/ou communautaire.

À noter, I'entrée en vigueur des règlements « Hygiène » au $1^{\mathrm{er}}$ janvier 2006.

Ainsi, la traçabilité étant un principe de base, qui doit être acquis, les opérateurs de la filière doivent être capables: d'appréhender et d'évaluer les risques inhérents à leur activité (détermination de « dangers » relatifs aux produits concernés), de prévenir les autorités compétentes en cas de non-conformité par rapport aux dispositions communautaires (ou nationales) spécifiques ou si un produit est jugé préjudiciable pour la santé (critère microbiologique ou risque émergent), et enfin être capables de mettre en œuvre des mesures de retrait ou rappel appropriées.

\section{La traçabilité est partie intégrante de la politique d'entreprise en matière de sécurité alimentaire}

Comme décrit précédemment, pour l'ensemble des sites Cargill de la division Grains \& Oilseeds Europe, une attention toute particulière est apportée au respect de la réglementation en matière de traçabilité, et ce depuis de nombreuses années.

Pour information, voici un extrait des principes directeurs de Cargill, Inc. à-propos de Food and
Product Safety : "Cargill is committed to providing safe food and feed for people and animals. We will use only those production, storage, and transportation facilities that ensure the safety of our products. We will choose our suppliers for their ability to meet our requirements for safe raw materials and ingredients. We will assist our external customers in developing customer solutions in the area of food safety. Management will provide the resources necessary for each and every employee to carry out their individual responsibilities to provide safe food and feed."

\section{Conclusion}

Tout comme Cargill, les opérateurs de l'ensemble de la filière des oléagineux n'ont pas attendu la nouvelle réglementation pour mettre en place et appliquer les obligations liées à la traçabilité.

S'il n'y a pas d'obligation de moyens, il y a cependant une obligation de résultats. Les opérateurs ont la responsabilité du choix des systèmes qu'ils mettent en place (déterminés en fonction d'une évaluation de risques et de leurs contraintes économiques) et doivent être en mesure de notifier aux autorités compétentes lorsqu'un danger est avéré.

Néanmoins, une meilleure collaboration entre les différents acteurs de la filière semble nécessaire, afin de répondre au mieux à ces exigences réglementaires en matière de sécurité alimentaire.

La mise en place de groupe de travail, incluant les fournisseurs de matières premières, les stockeurs, les transporteurs, les transformateurs et les distributeurs est nécessaire afin de :

- Faire évoluer les guides de bonnes pratiques existants et/ou pour en développer de nouveaux.

- Faire remonter au niveau des autorités compétentes et de la Commission, les difficultés d'interprétation, d'application ou les incohérences réglementaires lorsqu'elles sont mises à jour. 